\title{
Search for Non-Standard Model Physics in Rare Decays at the Tevatron
}

\author{
G. Volpi ${ }^{\mathrm{a}}$ on behalf of CDF and DØ collaborations \\ ${ }^{a}$ Univ and INFN Pisa, L.go Bruno Pontecorvo 3, 56127 Pisa, Italy
}

In this proceeding we report the most recent results from the CDF and D $\varnothing$ collaborations on the rare decays of b-mesons. The presented results show a new D $\varnothing$ limit for $\mathcal{B}\left(B_{s}^{0} \rightarrow \mu^{+} \mu^{-}\right)$that represent the best single measurement on this mode so far, an update of the $\mathcal{B}\left(B_{s}^{0} \rightarrow \phi \phi\right)$ with, for the first time, an angular analysis on the final state, a new measurement of the $\mathcal{B}\left(B^{0} \rightarrow K^{\star 0} \mu^{+} \mu^{-}\right)$and $\mathcal{B}\left(B^{+} \rightarrow K^{+} \mu^{+} \mu^{-}\right)$with an interesting measurement on the forward-backward asymmetry for those channels and the first observation of the $\mathcal{B}\left(B_{s}^{0} \rightarrow \phi \mu^{+} \mu^{-}\right)$.

\section{Introduction}

The search for the rare $b$-hadron decays is an important precision test for the Standard Model (SM). The decay modes described in this paper are all forbidden at the tree level in the SM and are only mediated by loop diagrams. BeyondSM (BSM) contributions are in general possible at tree level in some models or can contribute to the loops, with an effect on the amplitudes of the processes and in other observables. For those decay modes the smallness of the branching ratio (BR) by itself contribute also to make the new physics (NP) contribution evident.

The two Tevatron experiments, CDF[1] and $\mathrm{D} \varnothing[2]$, benefit of the large heavy-flavor production cross-section for $p \bar{p}$ collisions at $\sqrt{s}=$ $1.96 \mathrm{TeV}$. These conditions make the experiments competitive with the B-factories results on the $B^{0}$ and $B^{ \pm}$modes, with the unique opportunity at the same time to investigate the rare decays of the $B_{s}^{0}$ or other b-hadrons. The decay modes in this document involve both leptonic final states, with the $B_{(s)}^{0} \rightarrow \mu^{+} \mu^{-}$and $B^{0(+)} \rightarrow h \mu^{+} \mu^{-}$, where $h$ stands for a meson, and purely hadronic modes as the $B_{s}^{0} \rightarrow \phi \phi$, giving a further example of the richness of the B-physics programs at the two experiments.

\section{2. $B_{s}^{0} \rightarrow \phi \phi$ branching ratio and polariza- tion}

The $B_{s}^{0} \rightarrow \phi \phi$ decay mode in the SM is dominated by the contribution coming from the pen- guin diagram, indeed suppressed in the SM. In the recent years the penguin mediated processes have gathered some attention due to discrepancies found between SM prediction and data at Bfactories. Furthermore this $B \rightarrow V V$ final state can be used to measure $\sin \left(2 \beta_{s}\right)$. The CDF experiment observed this decay mode for the first time in 2005, with a $\mathrm{BR}$ of $\mathcal{B}\left(B_{s}^{0} \rightarrow \phi \phi\right)=$ $[1.4 \pm 0.6 \pm 0.6] \times 10^{-5}$, based on $180 \mathrm{pb}^{-1}[3]$.

The current update is based on a sample of $2.9 \mathrm{fb}^{-1}$. The online selection is made possible by two dedicated hardware systems XFT[4] and SVT[5]. These two systems, at the first ad second level of the trigger, are able to reconstruct the track parameters with high precision in about $20 \mu s$; in particular SVT uses the silicon information and allows to recognize events with tracks coming from a displaced vertex and with helix parameters compatible with a B-hadron decay.

The basic trigger selection used at the Level2 requires events having two tracks with $p_{T} \geq$ $2 \mathrm{GeV} / \mathrm{c}$ and $120 \mu \mathrm{m} \leq d_{0} \leq 1.0 \mathrm{~mm}$. The two trigger tracks must have an opening angle $2^{\circ} \leq|\Delta \theta| \leq 90^{\circ}$ in the transverse plane. The basic selection has minor changes as function of the instantaneous luminosity to keep the the trigger rate under control. The selection at Level-3 confirms the previous cuts and requires a transverse decay length $L_{x y} \geq 200 \mu \mathrm{m}$, where the distance is corrected by the beamline position.

The analysis cuts are optimized to maximize the standard figure of merit $F=S / \sqrt{S+B}$, where $S$ (B) represents the number of signal 
(background) events. The background is selected from the sidebands, while the signal is simulated using the CDF detailed Montecarlo (MC). As reference mode for the $\mathrm{BR}$, and control sample for the angular analysis, the $B_{s}^{0} \rightarrow J / \psi \phi$ is chosen. The reference mode selection is performed using the same trigger selection, the events in common with the di-muon trigger selection used in another analysis about $B_{s}^{0} \rightarrow J / \psi \phi[6]$ are vetoed to have a completely independent sample in order to check the results.

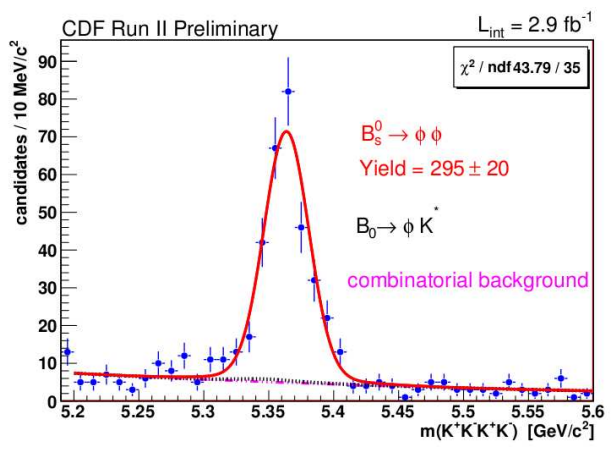

Figure 1. Invariant mass in four kaons hypothesis, the lines show the projection of the ML fit to extract the $B_{s}^{0} \rightarrow \phi \phi \mathrm{BR}$. The solid line represents the projection of the complete model, the black dotted line the peaking $B^{0} \rightarrow \phi K^{\star}$ contribution, and the violet dashed line the combinatorial background.

For the BR measurement the selected events are fitted using an unbinned maximum likelihood (ML) fit on the invariant mass of the four kaons, with a model composed of two gaussians for the signal peak, a peaking background to account for the $B^{0} \rightarrow \phi K^{\star}$ contribution, and a combinatorial background. From the fit results is shown in fig. 1, the number of signal events is $295 \pm 20$, that, correcting for the relative efficiency and using the world average value for $\mathcal{B}\left(B_{s}^{0} \rightarrow J / \psi \phi\right)$, give[7]:

$\mathcal{B}\left(B_{s}^{0} \rightarrow \phi \phi\right)=[2.40 \pm 0.21 \pm 0.86] \times 10^{-5}$

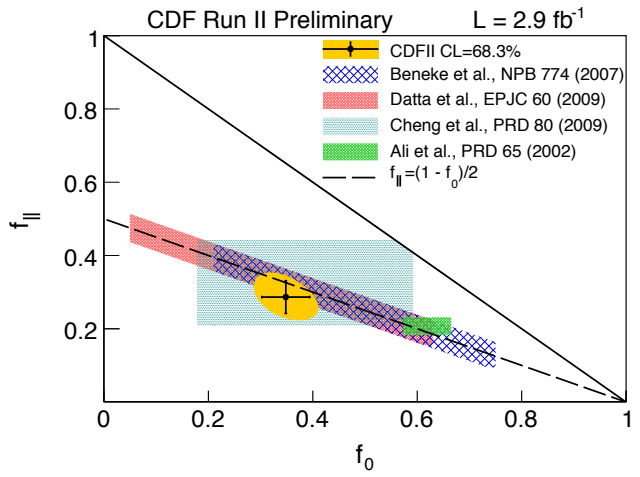

Figure 2. Comparison between the CDF measurement and some theoretical prediction.

Performing the fit with the addition of three angles, in the so called helicity reference frame, it is possible to disentangle the $\mathrm{CP}$ even and odd components. The fit method was cross checked with the $B_{s}^{0} \rightarrow J / \psi \phi$ mode, obtaining results compatible with the independent sample used in the $\sin \left(2 \beta_{s}\right)$ analysis[6]. The angular analysis here is time integrated, the $B_{s}^{0}$ time evolution is described using the values $1 / \Gamma_{L}=$ $1.408_{-0.030}^{+0.033} \mathrm{ps}, 1 / \Gamma_{H}=1.543_{-0.060}^{+0.058} \mathrm{ps}$ and the $\Delta \Gamma_{s}=0.062_{-0.037}^{+0.034} \mathrm{ps}^{-1}, \phi_{s}$ is set to 0 . In the fit the flavor tagging is not used, so it is not possible to separate $B_{s}^{0}$ from $\bar{B}_{s}^{0}$.

In the angular analysis the effect of the trigger acceptance on angular distribution of the CPeven and $\mathrm{CP}$-odd modes are taken into account using the CDF MC.

The angular analysis allowed to separate the longitudinal and transverse contribution, with a result of $f_{L}=0.348 \pm 0.041 \pm 0.021$ and $f_{T}=0.652 \pm 0.041 \pm 0.021[8]$. Fig. 2 shows a comparison between the experimental results and some predictions obtained using particular models. The comparison with the theoretical predictions shows a reasonable agreement with some of the models. 


\section{3. $B \rightarrow h \mu^{+} \mu^{-}$analysis}

The Flavor Changing Neutral Current (FCNC) transition $b \rightarrow s l^{+} l^{-}$in the $\mathrm{SM}$ is possible in penguin and box diagrams. The CDF analysis was performed on three different modes: $B^{0} \rightarrow$ $K^{\star 0} \mu^{+} \mu^{-}, B^{+} \rightarrow K^{+} \mu^{+} \mu^{-}$, and $B_{s}^{0} \rightarrow \phi \mu^{+} \mu^{-}$. The modes involving $B^{+}$and $B^{0}$ were already observed and studied at the B-Factories, the third mode was never observed before and only an upper limit to its BR exists[12,13].

The most recent CDF analysis uses $4.4 \mathrm{fb}^{-1}$ of data. The trigger selection requires the identification of two charged muons, with opposite charge, in the region with $|\eta| \leq 1$ and transverse momentum $p_{T}>1.5 \mathrm{GeV} / \mathrm{c}$ or $p_{T}>2.0 \mathrm{GeV} / \mathrm{c}$ according to the trigger condition. Other refinements on the selection are applied to keep the trigger rate under control. The kaon identification relies on the possibility to measure the energy deposited in the tracking chamber and the time-of-flight; the muon identification adds the information coming from the muon chambers combined in an likelihood.

The BR measurement is performed selecting for each $B_{q} \rightarrow h \mu^{+} \mu^{-}$the $B_{q} \rightarrow h J / \psi$ mode as reference, where $q$ stands for $u, d$, or $s$ quark and $h$ for $K^{+}, K^{\star}$, or $\phi$ meson. For each channel the cuts are optimized in two steps: the first optimization step selects the cuts to have the best significance with respect to the reference mode, in this first step the cuts are just rectangular cuts: this is the loose selection; in the second step the selection is chosen to have the best significance on the signal modes. Here an artificial neural network output variable is used: this is the $N N$ selection. In the final optimization there is a small difference between modes already observed and the $B_{s}^{0} \rightarrow \phi \mu^{+} \mu^{-}$: the $B^{+}$and $B^{0}$ modes were optimized to obtain the best significance, maximizing the figure of merit $S / \sqrt{S+B}$, for $B_{s}^{0}$ mode was instead used the figure of merit $S /(5 / 2+\sqrt{B})$ because the mode wasn't observed before and the aim was to obtain a statistical significance of $5 \sigma$.

The final BR indeed can be evaluated using the general formula:

$$
\frac{\mathcal{B}(\text { sgn })}{\mathcal{B}(\text { ref })}=\frac{N_{\text {sgn }}^{N N}}{N_{\text {ref }}^{L}} \frac{\epsilon_{\text {ref }}^{L}}{\epsilon_{\text {sgn }}^{L}} \frac{1}{\epsilon_{\text {sgn }}^{N N}} \times \mathcal{B}\left(J / \psi \rightarrow \mu^{+} \mu^{-}\right)
$$

where $N_{\operatorname{sgn}(r e f)}^{N N(L)}$ is the number of events for the signal (reference) after NN (loose) selection, $\epsilon_{\text {sgn(ref) }}^{L}$ is the signal (reference) efficiency for the loose cuts, and $\epsilon_{s g n}^{N N}$ is the signal efficiency after the NN selection.

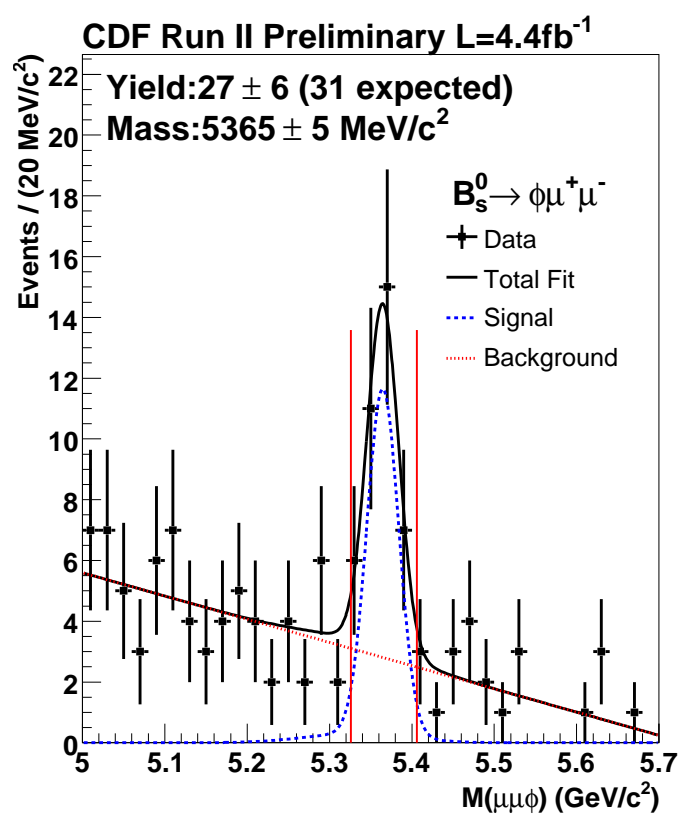

Figure 3. Mass projection for the fit of the $B_{s}^{0} \rightarrow$ $\phi \mu^{+} \mu^{-}$decay mode.

The results give:

$$
\begin{aligned}
\mathcal{B}\left(B^{+} \rightarrow K^{+} \mu^{+} \mu^{-}\right) & =[0.38 \pm 0.05 \pm 0.03] \times 10^{-6} \\
\mathcal{B}\left(B^{0} \rightarrow K^{\star} \mu^{+} \mu^{-}\right) & =[1.06 \pm 0.14 \pm 0.09] \times 10^{-6} \\
\mathcal{B}\left(B_{s}^{0} \rightarrow \phi \mu^{+} \mu^{-}\right) & =[1.44 \pm 0.33 \pm 0.46] \times 10^{-6}
\end{aligned}
$$

the statistical signal significance for the modes are: $8.5 \sigma, 9.7 \sigma$, and $6.3 \sigma$, with the first observation for the $B_{s}^{0} \rightarrow \phi \mu^{+} \mu^{-}$mode. Fig. 3 show the fit results for the $B_{s}^{0} \rightarrow \phi \mu^{+} \mu^{-}$mode. 


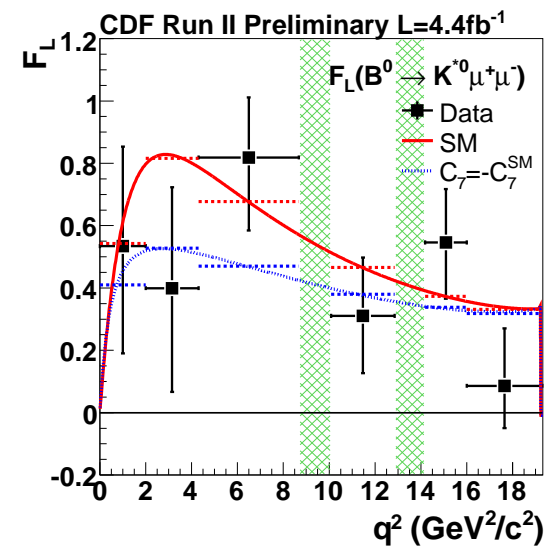

Figure 4. Longitudinal polarization of the $B^{0} \rightarrow$ $K^{\star} \mu^{+} \mu^{-}$in bins of di-muon invariant mass. The lines show the SM prediction and a NP model.

For the $B^{+(0)} \rightarrow K^{+(\star 0)} \mu^{+} \mu^{-}$modes it is also possible to extract other parameters of the decays, related to the kinematics, that are well predicted by the SM. Those parameters are the longitudinal polarization fraction (FL) and the forward-backward asymmetry (AFB).

The AFB and $K^{* 0} \mathrm{FL}$ are extracted from $\cos \theta_{\mu}$ and $\cos \theta_{K}$ distributions, respectively, where $\theta_{\mu}$ is the helicity angle between $\mu^{+}\left(\mu^{-}\right)$direction and the opposite of the $B(\bar{B})$ direction in the di-muon rest-frame, and $\theta_{K}$ is the angle between the kaon direction and the direction opposite to the $\mathrm{B}$ meson in the $K^{\star 0}$ rest frame.

The FL parameter is well predicted by the SM as function of the squared di-muon mass $q^{2}$. The result is shown in fig. 4 where within the statistical uncertainty the data are in agreement with the SM.

For both $\quad B^{0} \rightarrow K^{\star 0} \mu^{+} \mu^{-} \quad$ and $B^{+} \rightarrow K^{+} \mu^{+} \mu^{-}$, in the reference frame where the two muons decay at rest, the observable $\cos (\theta)$ is defined. The $\cos (\theta)$ variable is the scalar product between the directions of the $\mu^{+}$ and the original $B$ meson candidate in this particular reference frame. Separating the sample between $\cos (\theta)>0$ from the $\cos (\theta)<0$ it is pos-

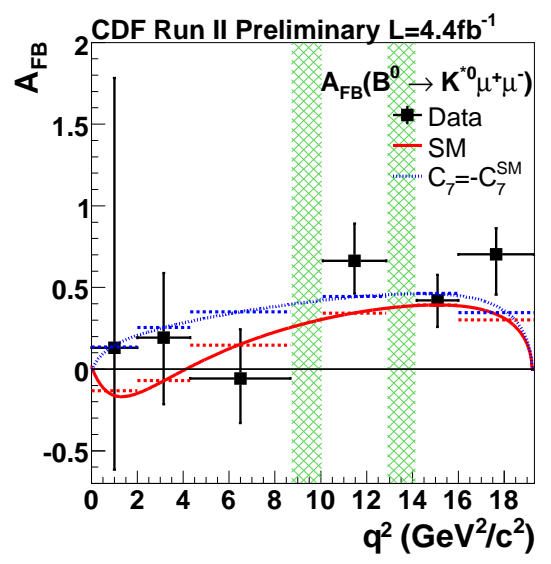

Figure 5. Result for the forward-backward asymmetry for the $B^{0} \rightarrow K^{\star 0} \mu^{+} \mu^{-}$in 6 bins of dimuon invariant mass. The two bands show the vetoes on the $J / \psi$ and $\psi^{\prime}$. The SM (the bottom solid red curve) and one NP model (the top blue dashed curve) predictions are compared with the experimental data.

sible to evaluate the so called forward-backward asymmetry:

$A_{F B}\left(q^{2}\right)=\frac{N\left(q^{2}, \cos (\theta)>0\right)-N\left(q^{2}, \cos (\theta)<0\right)}{N\left(q^{2}, \cos (\theta)>0\right)+N\left(q^{2}, \cos (\theta)<0\right)}$

The value of the asymmetry is predicted in the SM and in many BSM theories as a function of the di-muon mass. Fig. 5 shows how the experimental data are in agreement with the SM prediction but also with some of the BSM models[9].

4. $B_{(s)}^{0} \rightarrow \mu^{+} \mu^{-}$search

The $B_{(s)}^{0} \rightarrow \mu^{+} \mu^{-}$decay is considered one of the golden channels to test the SM prediction in the flavor sector. This is because the purely leptonic final state limits the uncertainty due to hadronic form factors. Moreover the contribution of the NP in many models can be precisely computed. In this scenario the SM prediction for the $\mathrm{BR}$ is $\mathcal{B}\left(B_{s}^{0} \rightarrow \mu^{+} \mu^{-}\right)=[3.6 \pm 0.4] \times 10^{-9}[10]$, with a further suppression $\left(V_{t d} / V_{t s}\right)^{2} \simeq 0.04$ for 
the $B^{0} \rightarrow \mu^{+} \mu^{-}$.

Those decay modes are also favorite from the experimental point of view, because the di-muon final state is a very clean signature. The search is performed by both CDF and D $\varnothing$ with a very similar selection, the main differences are the muon coverage and mass resolution: CDF has a good coverage up to $|\eta|<1$ with a mass resolution of about $25 \mathrm{MeV} / \mathrm{c}^{2}$, DØ coverages is up to $|\eta|<3$ with a mass resolution about $100 \mathrm{MeV} / \mathrm{c}^{2}$.

The data selection and the analysis technique are similar in both experiments: the selection is based on a search for a di-muon candidate, with a multivariate classifier to identify $B_{(s)}^{0} \rightarrow$ $\mu^{+} \mu^{-}$candidate. The CDF analysis uses an artificial neural network (ANN), while D $\varnothing$ uses a Bayesian neural network (BNN). The classifier uses as input variable the muon momenta, the candidate isolation, the decay length and other kinematic variables, with the exclusion of the dimuon mass. The background training sample is taken from the sidebands while the signal sample is obtained generating $B_{s}^{0} \rightarrow \mu^{+} \mu^{-}$events with an event generator and processing the events through the experiment MC simulation. The CDF experiment, due to the better mass resolution, can also perform a direct search for the $B^{0} \rightarrow \mu^{+} \mu^{-}$decay mode.

The background sources are semileptonic decays of $b$ and $c$-hadron, in particular there are two sources: one is due to the double semileptonic decays, like $B \bar{B} \rightarrow \mu^{+} \mu^{-} X$ or $D \bar{D} \rightarrow \mu^{+} \mu^{-} X$, the other to sequential semileptonic decays, like $B \rightarrow \mu^{+} \nu \bar{D}, \bar{D} \rightarrow \mu^{-} \bar{\nu} X$. An additional source to the previous is when there is a real muon and a fake muon from misidentification of pions and kaons. A peaking background comes from the hadronic decays $B \rightarrow h^{+} h^{\prime-}$, when both hadrons are faked as muons, but this is found to be negligible for the $B_{s}^{0} \rightarrow \mu^{+} \mu^{-}$.

The BR of the decay is obtained using as reference the $B^{+} \rightarrow J / \psi K^{+}$decay mode using the following formula:

$\mathcal{B}\left(B_{s}^{0} \rightarrow \mu^{+} \mu^{-}\right)=\frac{N\left(B_{s}^{0}\right)}{N\left(B^{+}\right)} \cdot \frac{\epsilon_{B^{+}}}{\epsilon_{B_{s}^{0}}} \cdot \frac{f_{u}}{f_{s}} \cdot \mathcal{B}\left(B^{+}\right)$

where $N\left(B_{s}^{0}\right)$ and $\epsilon_{B_{s}^{0}}$ are the yield and the efficiency for the signal, $N\left(B^{+}\right)$and $\epsilon_{B^{+}}$are the
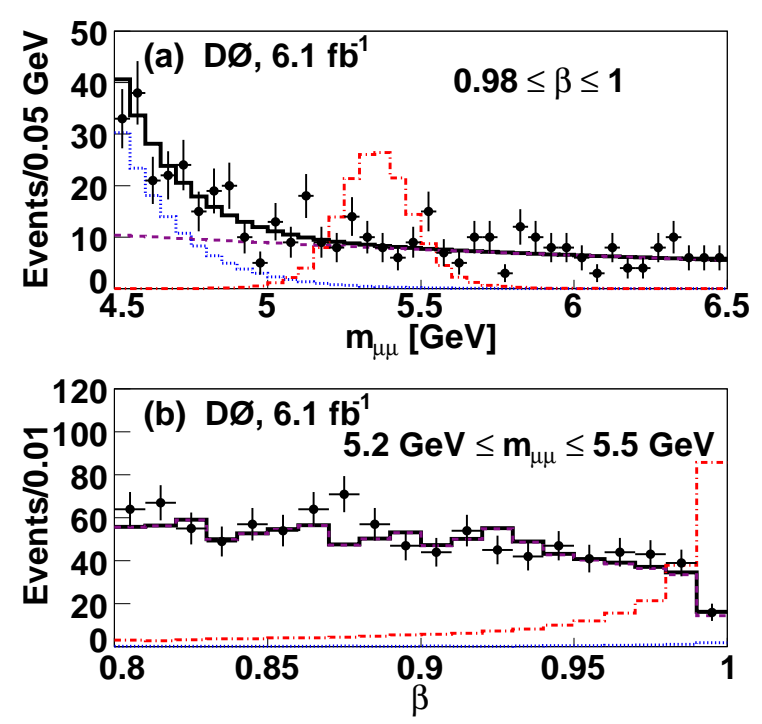

Figure 6. Mass projection from the $\mathrm{D} \varnothing$ analysis for the most significant bin for the BNN output (on the top), and for the BNN in the $B_{s}^{0}$ mass window (on the bottom). The red curve represents the the $B_{s}^{0}$ signal multiplied by a factor 10 , the other curves the combinatorial and fake muons background.

number of $B^{+}$decay mode and the analysis efficiency. For the fragmentation function $\mathrm{D} \varnothing$ chooses the PDG 2006 value $f_{u} / f_{s}=3.86 \pm 0.59$ to better compare with the previous measurements. The limit is evaluated using the pseudofrequentist confidence level approach $\left(\mathrm{CL}_{\mathrm{s}}\right)[14]$. $\mathrm{D} \varnothing$ based on $6.1 \mathrm{fb}^{-1}$ doesn't observe any excess, setting an upper limit to $\mathcal{B}\left(B_{s}^{0} \rightarrow \mu^{+} \mu^{-}\right)<$ $5.1 \times 10^{-8}$ at the $95 \%$ C.L. shown in fig. 6 [15]. This is consistent with the CDF preliminary result published in 2009 , based on $3.7 \mathrm{fb}^{-1}$, already presented at conferences, with the limit $\mathcal{B}\left(B_{s}^{0} \rightarrow \mu^{+} \mu^{-}\right)<4.3 \times 10^{-8}$ at $95 \%$ C.L. [16].

\section{Conclusions}

The Tevatron experiments are giving a very important contribution in the study of $B$ rare decays, helping to define how the new physics could 
appear in this field. The CDF experiment reports a new results for the the $B_{s}^{0} \rightarrow \phi \phi$ mode, with the first determination of the polarization amplitudes, a measurement on the $B^{0(+)} \rightarrow h \mu^{+} \mu^{-}$ modes with a precision similar to the one obtained at the B-factories on the same modes, and the first observation of the $B_{s}^{0} \rightarrow \phi \mu^{+} \mu^{-}$. The $\mathrm{D} \varnothing$ experiment reports an update on the search of the $B_{s}^{0} \rightarrow \mu^{+} \mu^{-}$decay. No events are found, the limit on this mode is compatible with the expectation and with the recent CDF measurement.

The presented results can be further improved in the middle term because they are generally based on a part of the total $10 \mathrm{fb}^{-1}$ per experiment that the Tevatron is expected to provide.

\section{REFERENCES}

1. The CDFII Detector Technical Design Report, Fermilab-Pub-96/390-E; D. Acosta, et al., Phys. Rev. D 71, 032001 (2005).

2. D0 Collaboration, V. M. Abazov et al., Nucl. Instrum. Methods in Phys. Res. A 565, 463 (2006).

3. D.Acosta et al. (CDF Collaboration),Phys. Rev. Lett. 95, 031801, 2005.

4. E. J. Thomson et al. Online Track Processor for the CDF Upgrade. IEEE Trans. Nucl. Sci., 49, 2002.

5. W. Ashmanskas, et al., Nucl. Instrum. Methods A 518, 532 (2004).

6. T. Aaltonen et al. [CDF Collaboration], Phys. Rev. Lett. 100 (2008) 161802 [arXiv:0712.2397 [hep-ex]].

7. CDF Collaboration, CDF-Note 10064 (2010).

8. CDF Collaboration, CDF-Note 10120(2010).

9. CDF Collboration, CDF-Note 10047 (2009).

10. A. J. Buras, Prog. Theor. Phys. 122, 145 (2009).

11. W.-M. Yao et al., Journal of Physics G 33, 1 (2006).

12. T. Aaltonen et al. (CDF), Phys. Rev. D79, 011104 (2009), arXiv:0804.3908.

13. V. M. Abazov et al. (D0), Phys. Rev. D74, 031107 (2006), arXiv:hep-ex/0604015.

14. T. Junk, Nucl. Instrum. Methods in Phys. Res. A 434, 435 (1999).

15. DØ Collaboration, FERMILAB-PUB-10-202-
E (2010).

16. CDF Collaboration, CDF Note 9892 (2009), arXiv:1006.3469 [hep-ex]. 